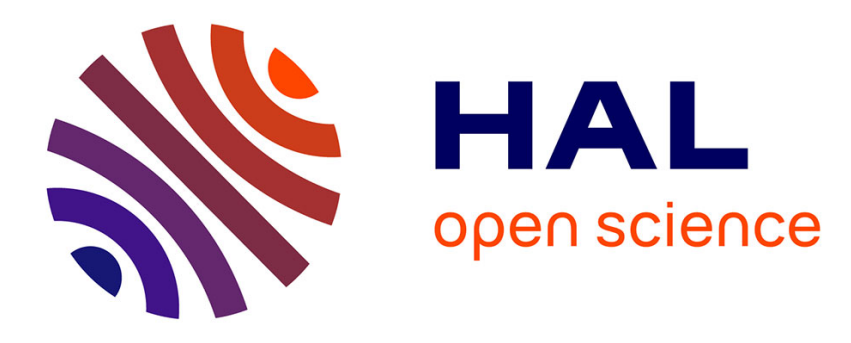

\title{
On exact controllability of linear time delay systems of neutral type
}

\author{
Rabah Rabah, Grigory M. Sklyar
}

\section{To cite this version:}

Rabah Rabah, Grigory M. Sklyar. On exact controllability of linear time delay systems of neutral type. J. Chiasson and J.J. Loiseau. Application of time delay systems, Springer, pp.165-171, 2007, Lecture Notes on Control and Information Systems. hal-00580066

\section{HAL Id: hal-00580066 https://hal.science/hal-00580066}

Submitted on 25 Mar 2011

HAL is a multi-disciplinary open access archive for the deposit and dissemination of scientific research documents, whether they are published or not. The documents may come from teaching and research institutions in France or abroad, or from public or private research centers.
L'archive ouverte pluridisciplinaire HAL, est destinée au dépôt et à la diffusion de documents scientifiques de niveau recherche, publiés ou non, émanant des établissements d'enseignement et de recherche français ou étrangers, des laboratoires publics ou privés. 



\title{
On exact controllability of linear time delay systems of neutral type
}

\author{
Rabah Rabah ${ }^{1}$ and Grigory Sklyar ${ }^{2}$ \\ 1 Institut de Recherche en Communications et Cybernétique de Nantes, École des \\ Mines de Nantes, 4 rue Alfred Kastler BP 2072244307 Nantes Cedex 3, France. \\ rabah@emn.fr \\ 2 Institute of Mathematics, University of Szczecin, Wielkopolska 15, 70451 \\ Szczecin, Poland. sklar@sus.univ.szczecin.pl
}

Summary. The problem of exact null controllability is considered for linear neutral type systems with distributed delay. A characterization of this problem is given. The minimal time of controllability is precised. The results are based on the analysis of the Riesz basis property of eigenspaces in Hilbert space. Recent results on the moment problem and properties of exponential families are used.

\section{Introduction}

The problem of controllability for delay systems was considered by several authors in different framework. One approach is based on the analysis of time delay system in a module framework (space over ring, see [8]). In this case the controllability problem is considered in a formal way using different interpretations of the Kalman rank condition. Another approach is based on the analysis of time delay systems in vector spaces with finite or infinite dimension. A powerful tool is to consider a delay system as a system in a Banach functional space, this approach was developed widely in [5]. Because the state space for delay systems is a functional space, the most important notion is the function space controllability. A first important contribution in the characterization of null functional controllability was given by Olbrot [10] by using some finite dimensional tools as $(A, B)$-invariant subspaces for an extended system. For retarded systems one can refer to [7] (and references therein) for the analysis of function space controllability in abstract Banach spaces. The case of neutral type systems with discrete delay was also considered in such a framework (see O'Connor and Tarn [9] and references therein). A general analysis of the time delay systems in infinite dimensional spaces is given in the book [3] where several methods and references are given. 
The problem considered in this paper is close to that studied in [9]. In this work the exact controllability problem was considered for neutral type systems with discrete delay using a semigroup approach in Sobolev spaces $W_{2}^{(1)}$ and a boundary control problem.

We consider the problem of controllability for distributed delay system of neutral type in the space $M_{2}\left(-h, 0 ; \mathbb{C}^{n}\right)=\mathbb{C}^{n} \times L_{2}\left(-h, 0 ; \mathbb{C}^{n}\right)$ which is natural for control problems. The semigroup theory developed here is based on the Hilbert space model introduced in [4]. One of our result is a generalization of the result in [9]. The main non trivial precision is the time of controllability. We generalize the results given [6] for the case of a single input and one localized delay (see also $[2,14]$ ). The approach developed here is different from that of [9]. Our main results are based on the characterization of controllability as a moment problem and using some recent results on the solvability of this problem (see [1] for the main tools used here). Using a precise Riesz basis in the space $M_{2}\left(-h, 0 ; \mathbb{C}^{n}\right)$ we can give a characterization of null-controllability and of the minimal time of controllability.

The present paper contains only the main idea of the approach and the formulations of the main results on exact controllability. A complete presentation of this approach is the subject of our extensive work which is to be published. One can also find the detailed proofs in the the preprint [11].

\section{The model and the controllability problem}

We study the following neutral type system

$$
\dot{z}(t)=A_{-1} \dot{z}(t-h)+\int_{-h}^{0} A_{2}(\theta) \dot{z}(t+\theta) \mathrm{d} \theta+\int_{-h}^{0} A_{3}(\theta) z(t+\theta) \mathrm{d} \theta+B u,
$$

where $A_{-1}$ is constant $n \times n$-matrix, $\operatorname{det} A_{-1} \neq 0, A_{2}, A_{3}$ are $n \times n$-matrices whose elements belong to $L_{2}(-h, 0), h>0$ is a constant delay. We consider the operator model of the neutral type system (1) introduced by Burns and al. in product spaces. The state space is $M_{2}\left(-h, 0 ; \mathbb{C}^{n}\right)=\mathbb{C}^{n} \times L_{2}\left(-h, 0 ; \mathbb{C}^{n}\right)$, shortly $M_{2}$, and (1) is rewritten as

$$
\frac{\mathrm{d}}{\mathrm{d} t}\left(\begin{array}{c}
y(t) \\
z_{t}(\cdot)
\end{array}\right)=\mathcal{A}\left(\begin{array}{c}
y(t) \\
z_{t}(\cdot)
\end{array}\right)+\mathcal{B} u
$$

where the operator $\mathcal{A}$ is given by

$$
\mathcal{A}\left(\begin{array}{c}
y(t) \\
z_{t}(\cdot)
\end{array}\right)=\left(\begin{array}{c}
\int_{-h}^{0} A_{2}(\theta) \dot{z}_{t}(\theta) \mathrm{d} \theta+\int_{-h}^{0} A_{3}(\theta) z_{t}(\theta) \mathrm{d} \theta \\
\mathrm{d} z_{t}(\theta) / \mathrm{d} \theta
\end{array}\right)
$$

with the domain

$$
\mathcal{D}(\mathcal{A})=\left\{(y, z(\cdot)): z \in H^{1}\left(-h, 0 ; \mathbb{C}^{n}\right), y=z(0)-A_{-1} z(-h)\right\} \subset M_{2} .
$$


The operator $\mathcal{A}$ is the infinitesimal generator of a $C_{0}$-group. The operator $\mathcal{B}$ is defined by $\mathcal{B} u=(B u, 0)$. The relation between the solutions of the neutral type system (1) and the system (2) is given by the substitutions

$$
y(t)=z(t)-A_{-1} z(t-1), \quad z_{t}(\theta)=z(t+\theta) .
$$

The reachability set at time $T$ is defined by

$$
\mathcal{R}_{T}=\left\{\int_{0}^{T} e^{\mathcal{A} t} \mathcal{B} u(t) d t: u(\cdot) \in L_{2}\left(0, T ; \mathbb{C}^{n}\right)\right\}
$$

It is easy to show that $\mathcal{R}_{T_{1}} \subset \mathcal{R}_{T_{2}}$ as $T_{1}<T_{2}$. An important result is that $\mathcal{R}_{T} \subset \mathcal{D}(\mathcal{A}) \subset M_{2}$. This non-trivial fact permits to formulate the nullcontrollability problem in the following setting:

i) To find maximal possible set $\mathcal{R}_{T}$ (depending on $T$ );

ii) To find minimal $T$ for which the set $\mathcal{R}_{T}$ becomes maximal possible, i.e. $\mathcal{R}_{T}=\mathcal{D}(\mathcal{A})$.

Definition 1. The system (2) is said null-controllable at the time $T$ if $\mathcal{R}_{T}=$ $\mathcal{D}(\mathcal{A})$

The main tool is to consider the null-controllability problem as a problem of moments.

\subsection{The moment problem}

In order to formulate the moment problem we need a Riesz basis in the Hilbert space $M_{2}$. We recall that a Riesz basis is a basis which may be transformed to an orthogonal basis with respect to another equivalent scalar product. Each Riesz basis possesses a biorthogonal basis. Let $\{\varphi\}$ be a Riesz basis in $M_{2}$ and $\{\psi\}$ the corresponding biorthogonal basis. Then for each $x \in M_{2}$ we have $x=\sum_{\varphi \in\{\varphi\}}\langle x, \psi\rangle \varphi$. In a separable Hilbert space there always exists a Riesz basis.

A state $x=\left(\begin{array}{c}y \\ z(\cdot)\end{array}\right) \in M_{2}$ is reachable at time $T$ by a control $u(\cdot) \in$ $L_{2}\left(0, T ; \mathbb{C}^{r}\right)$ iff the steering condition

$$
x=\left(\begin{array}{c}
y \\
z(\cdot)
\end{array}\right)=\int_{0}^{T} \mathrm{e}^{\mathcal{A} t} \mathcal{B} u(t) \mathrm{d} t .
$$

holds. This steering condition may be expanded using the basis $\{\varphi\}$. A state $x$ is reachable iff

$$
\sum_{\varphi \in\{\varphi\}}\langle x, \psi\rangle \varphi=\sum_{\varphi \in\{\varphi\}} \int_{0}^{T}\left\langle\mathrm{e}^{\mathcal{A} t} \mathcal{B} u(t), \psi\right\rangle \mathrm{d} t \varphi
$$


for some $u(\cdot) \in L_{2}\left(-h, 0 ; \mathbb{R}^{r}\right)$. Then the steering condition (3) can be substituted by the following system of equalities

$$
\langle x, \psi\rangle=\int_{0}^{T}\left\langle\mathrm{e}^{\mathcal{A} t} \mathcal{B} u(t), \psi\right\rangle \mathrm{d} t, \quad \psi \in\{\psi\} .
$$

Let $\left\{b_{1}, \ldots, b_{r}\right\}$ be an arbitrary basis in $\operatorname{Im} B$, the image of the matrix $B$ and $\mathbf{b}_{i}=\left(\begin{array}{c}b_{i} \\ 0\end{array}\right) \in M_{2}, i=1, \ldots, r$. Then the right hand side of (4) takes the form

$$
\int_{0}^{T}\left\langle\mathrm{e}^{\mathcal{A} t} \mathcal{B} u(t), \psi\right\rangle \mathrm{d} t=\sum_{i=1}^{r} \int_{0}^{T}\left\langle\mathrm{e}^{\mathcal{A} t} \mathbf{b}_{i}, \psi\right\rangle u_{i}(t) \mathrm{d} t .
$$

Effectiveness of the proposed approach becomes obvious if we assume that the operator $\mathcal{A}$ possess a Riesz basis of eigenvector. This situation is characteristic, for example, for control systems of hyperbolic type when $\mathcal{A}$ is skew-adjoint $\left(\mathcal{A}^{*}=-\mathcal{A}\right)$ and has a compact resolvent (see, for example, [1], [16], [17]). Let in this case $\left\{\varphi_{k}\right\}, k \in \mathbb{N}$, be a orthonormal eigenbasis with $\mathcal{A} \varphi_{k}=i \lambda_{k} \varphi_{k}$, $\lambda_{k} \in \mathbb{R}$. Assuming for simplicity $r=1, b_{1}=b=\sum_{k} \alpha_{k} \varphi_{k}, \alpha_{k} \neq 0$, we have from (4), (5)

$$
\frac{x_{k}}{\alpha_{k}}=\int_{0}^{T} e^{-i \lambda_{k} t} u(t) d t, \quad k \in \mathbb{N},
$$

where $x=\sum_{k} x_{k} \varphi_{k}$. Equalities (6) are a non-Fourier trigonometric moment problem whose solvability is closely connected with the property for the family of exponentials $e^{-i \lambda_{k} t}, k \in \mathbb{N}$, to form a Riesz basis on the interval $[0, T]([1])$. In particular, if $e^{-i \lambda_{k} t}$ forms a Riesz basis of $L_{2}\left[0, T_{0}\right]$ then one has

$$
\mathcal{R}_{T}=\left\{x: \sum_{k}\left(\frac{x_{k}}{\alpha_{k}}\right)^{2}<\infty\right\} \text { for all } T \geq T_{0} .
$$

Obviously formula (7) gives the complete answer to the both items of the controllability problem. Returning now to neutral type systems we observe that the operator $\mathcal{A}$ given in (2) is not skew-adjoint and, moreover, does not possess a basis even of generalized eigenvectors. So the choice of a proper Riesz basis in context of formulas (4), (5) is an essentially more complicated problem.

\subsection{The choice of basis}

In order to design the needed basis for our case we use spectral the properties of the operator $\mathcal{A}$ obtained in [13]. Let $\mu_{1}, \ldots, \mu_{\ell}, \mu_{i} \neq \mu_{j}$ be eigenvalues of $A_{-1}$ and let the integers $p_{m}$ be defined as : $\operatorname{dim}\left(A_{-1}-\mu_{m} I\right)^{n}=p_{m}, m=$ $1, \ldots, \ell$. Denote by

$$
\lambda_{m}^{(k)}=\frac{1}{h}\left(\ln \left|\mu_{m}\right|+\mathrm{i}\left(\arg \mu_{m}+2 \pi k\right)\right), m=1, \ldots, \ell ; k \in \mathbb{Z},
$$


and let $L_{m}^{(k)}$ be the circles of the fixed radius $r \leq r_{0}=\frac{1}{3} \min \left|\lambda_{m}^{(k)}-\lambda_{i}^{(j)}\right|$ centered at $\lambda_{m}^{(k)}$.

Let $\left\{V_{m}^{(k)}\right\}_{\substack{k \in \mathbb{Z} \\ m=1, \ldots, \ell}}$ be a family of $\mathcal{A}$-invariant subspaces given by

$$
V_{m}^{(k)}=P_{m}^{(k)} M_{2}, \quad P_{m}^{(k)}=\frac{1}{2 \pi \mathrm{i}} \int_{L_{m}^{(k)}} R(\mathcal{A}, \lambda) d \lambda .
$$

The following theorem plays an essential role in our approach

Theorem 1. [12] There exists $N_{0}$ large enough such that for any $N \geq N_{0}$ i) $\operatorname{dim} V_{m}^{(k)}=p_{m}, k \geq N$,

ii) the family $\left\{V_{m}^{(k)}\right\}_{\substack{|k| \geq N \\ m=1 \ldots, \ell}} \cup \widehat{V}_{N}$ forms a Riesz basis (of subspaces) in $M_{2}$, where $\widehat{V}_{N}$ is a finite-dimensional subspace $\left(\operatorname{dim} \widehat{V}_{N}=2(N+1) n\right)$ spanned by all generalized eigenvectors corresponding to all eigenvalues of $\mathcal{A}$ located outside of all circles $L_{m}^{(k)},|k| \geq N, m=1, \ldots, \ell$.

Using this theorem we construct a Riesz basis $\{\varphi\}$ of the form

$$
\left\{\varphi_{m, j}^{k},|k|>N ; m=1, \ldots, l ; j=1, \ldots, p_{m}\right\} \cup\left\{\hat{\varphi}_{j}^{N}, j=1, \ldots, 2(N+1) n\right\}
$$

where for any $m=1, \ldots, l$, and $k:|k|>N$ the collection $\left\{\varphi_{m, j}^{k}\right\}_{j=1, \ldots, p_{m}}$ is in a special way chosen basis of $V_{m}^{(k)}$ and $\left\{\hat{\varphi}_{j}^{N}\right\}_{j=1, \ldots, 2(N+1) n}$ is a basis of $\hat{V}_{N}$. In this basis equalities (4) with regard to (5) turns into a moment problem with respect to a special collection of quasipolynomials. Analyzing the mentioned moment problem by means of the methods given in [1] we obtain our main results concerning the null-controllability problem.

\section{The main results}

The characterization of the null-controllability is given by the following Theorem.

Theorem 2. The system (2) is null-controllable by controls from $L_{2}(0, T)$ for some $T>0$ iff the following two conditions hold:

i) $\operatorname{rank}\left[\Delta_{\mathcal{A}}(\lambda) \quad B\right]=n, \quad \forall \lambda \in \mathbb{C}$; where

$$
\Delta_{\mathcal{A}}(\lambda)=-\lambda I+\lambda \mathrm{e}^{-\lambda h} A_{-1}+\lambda \int_{-h}^{0} \mathrm{e}^{\lambda s} A_{2}(s) \mathrm{d} s+\int_{-h}^{0} \mathrm{e}^{\lambda s} A_{3}(s) \mathrm{d} s .
$$

ii) $\operatorname{rank}\left[\begin{array}{llll}B & A_{-1} B & \cdots & A_{-1}^{n-1} B\end{array}\right]=n$.

The main results on the time of controllability are as follows.

Theorem 3. Let the conditions i) and ii) of Theorem 2 hold. Then

i) The system (2) is null-controllable at the time $T$ as $T>n h$; 
ii) If the system (2) is of single control $(r=1)$, then the estimation of the time of controllability in i) is exact, i.e. the system is not controllable at time $T=n h$.

For the multivariable case, the time depends on some controllability indices. suppose that $\operatorname{dim} B=r$. Let $\left\{b_{1}, \ldots, b_{r}\right\}$ be an arbitrary basis noted $\beta$. Let us introduce a set integers. We denote by $B_{i}=\left(b_{i+1}, \ldots, b_{r}\right), i=0,1, \ldots, r-1$, which gives in particular $B_{0}=B$ and $B_{r-1}=\left(b_{r}\right)$ and we put formally $B_{r}=0$. Let us consider the integers

$$
n_{i}^{\beta}=\operatorname{rank}\left[\begin{array}{llll}
B_{i-1} & A_{-1} B_{i-1} & \cdots & A_{-1}^{n-1} B_{i-1}
\end{array}\right], \quad i=1, \ldots, r,
$$

corresponding to the basis $\beta$. We need in fact the integers

$$
m_{i}^{\beta}=n_{i-1}^{\beta}-n_{i}^{\beta} \text {, }
$$

Let us denote by

$$
m_{\min }=\max _{\beta} m_{1}^{\beta} \quad m_{\max }=\min _{\beta} \max _{i} m_{i}^{\beta},
$$

for all possible choice of a basis $\beta$.

The main result for the multivariable case is the following Theorem.

Theorem 4. Let the conditions i) and ii) of the Theorem 2 hold, then

i) The system (2) is null-controllable at the time $T>m_{\max } h$;

ii) The system (2) is not null-controllable at the time $T<m_{\min } h$.

The proofs are based on the construction of a special Riesz basis of $\mathcal{A}$ invariant subspaces in the space $M_{2}$ according to [12] and on the analysis of the properties of some quasi-exponential functions to be a Riesz basis in $L_{2}(0, T)$ depending of the time $T[1]$.

\section{Final Conclusions}

For the delayed system of neutral type (1) we have the following results:

i) All the reachable states $z(t), t \in[T-1, T]$ from 0 are elements of $H^{1}[T-1, T]$ (independently of $T$ ).

ii) If $T>m_{\max } h$ then the set of reachable states on $[0, T]$ coincides with $H^{1}[T-1, T]$.

iii) If $T<m_{\min } h$ then the set of reachable states is an essential subspace of $H^{1}[T-1, T]$.

If $r=1$, this gives $m_{\max }=m_{\min }=n$ and then

iv) For $T=n$ the reachable states form a subspace in $H^{1}[T-1, T]$ of finite codimension; for $T<n$ there exists an infinite-dimensional subspace in $H^{1}[T-1, T]$ of unreachable states.

Acknowledgements. This work was realized with the partial financial support of the French-Polish grant Polonium No 07599VH. 


\section{References}

1. Avdonin S. A., Ivanov S. A., Families of exponentials. The method of moments in controllability problems for distributed parameter systems. Cambridge University Press, Cambridge, 1995.

2. Banks H. T., Jacobs Marc Q., Langenhop C. E., Characterization of the controlled states in $W_{2}^{(1)}$ of linear hereditary systems. SIAM J. Control, Vol. 13, No, May 1975.

3. Bensoussan A., Da Prato G., Delfour M. C.; Mitter S. K. Representation and control of infinite-dimensional systems. Vol. 1. Systems \& Control: Foundations \& Applications. Birkhäuser Boston, Inc., Boston, MA, 1992. xiv+315 pp.

4. Burns, John A., Herdman, Terry L., Stech, Harlan W. Linear functionaldifferential equations as semigroups on product spaces. SIAM J. Math. Anal., 14 (1983), no. 1, pp. 98-116.

5. Hale J. and Verduyn Lunel S. M, Theory of functional differential equations, Springer-Verlag, New York, 1993.

6. Jacobs M. Q., Langenhop C. E., Criteria for function space controllability of linear neutral systems. SIAM J. Control and Optimization, vol. 14, No 6, 1976, pp. 1009-1048.

7. Manitius A. and Triggiani R., Function space controllability of linear retarded systems: A derivation from abstract operator conditions, SIAM J. on Control and Optimization, vol. 16, 1978, pp. 599-645.

8. Morse A. S., Ring models for delay differential equation. Automatica, vol. 12, pp. 529-531.

9. O'Connor, D. A. and Tarn, T. J. On the function space controllability of linear neutral systems. SIAM J. Control Optim. 21 (1983), no. 2, 306-329.

10. Olbrot A. W., On degeneracy and related problems for linear time lag systems. Ricerche di Automatica, vol. 3, pp. 203-220

11. Rabah R., Sklyar G. M. The analysis of exact controllability of neutral type systems by the moment problem approach. Preprint IRCCyN/École des Mines de Nantes, No 8, Juillet 2005.

12. Rabah R., Sklyar G. M. and Rezounenko A. V., Generalized Riesz basis property in the analysis of neutral type systems, C.R. Acad. Sci. Paris, Ser.I, 337(2003), pp. 19-24.

13. Rabah R., Sklyar G. M. and Rezounenko A. V., Stability analysis of neutral type systems in Hilbert space. J. Differential Equations, Vol 214, Issue 2, 2005, 391-428.

14. Rodas Hernan Rivera, C. E. Langenhop, A sufficient condition for function space controllability of a linear neutral system. SIAM J. Control and Optimization, Vol. 16, No 3, May 1978, pp. 429-435. 